\title{
Design and Implementation of the Home-Based Cooperative Cache for PVFS
}

\author{
In-Chul Hwang, Hanjo Jung, Seung-Ryoul Maeng, and Jung-Wan Cho \\ Division of Computer Science, Dept. of Electrical Engineering \& Computer Science, \\ KAIST, 373-1 Kusung-dong Yusong-gu, Taejon, 305-701, Republic of Korea \\ \{ichwang, hanjo, maeng, jwcho\}@calab.kaist.ac.kr
}

\begin{abstract}
Recently, there has been much research about cluster computing to get high performance using low-cost PCs connected with high-speed interconnection networks. In many research areas, many distributed file systems have been developed. In many distributed file systems, PVFS (Parallel Virtual File System) provides users with high bandwidth by stripping data over I/O servers. In PVFS, there is no file system cache. For a new file system cache for PVFS, we designed and implemented cooperative cache for PVFS (CoopcPVFS). Because the previous developed Coopc-PVFS is a hint-based cooperative cache, a cache manager reads/writes files using approximately correct information so that it has a little read/write overhead. And previous studies about cooperative cache are only focused on shared read data and don't care about write performance. In this paper, we describe the design and implementation of the home-based Coopc-PVFS to improve read/write performance. Also, we evaluate and analysis the performance of the homebased Coopc-PVFS in comparison to PVFS and to the hint-based Coopc-PVFS.
\end{abstract}

\section{Introduction}

Recently, there has been much research about cluster computing to get high performance using low-cost PCs connected with high-speed inter-connection networks. For an efficient usage of cluster computing, many efficient components of cluster is necessary - efficient interconnection networks, Operating System supports and etc. Among many research areas, many distributed file systems which access disks slower than any other component in cluster computing have been developed.

Among research areas of distributed file system, cooperative cache $[3,4,5]$ was proposed to reduce servers' load and to get high performance. Because the access time of other clients' memories is faster than that of servers' disks, to get a block from other clients' memories is faster than to get the block from servers' disk. In cooperative cache, a client finds a block first from its own file system cache, and then other clients' file system caches before getting the block from servers' disks.

In many distributed file systems, PVFS (Parallel Virtual File System) [1] provides users with high bandwidth by stripping data over $\mathrm{I} / \mathrm{O}$ servers. There is no file system cache in PVFS - PVFS just supports data transfers from/to I/O servers. For a new file system cache for PVFS, we designed and implemented cooperative cache for PVFS (Coopc-PVFS) [9]. 
Because the previous developed Coopc-PVFS is a hint-based cooperative cache, a cache manager reads/writes files using approximately correct information. If a hint is incorrect, read/write overhead can be large. And previous studies about cooperative cache are only focused on shared read data and don't care about write performance. To solve this problem, we design and implement the home-based Coopc-PVFS. In the home-based Coopc-PVFS, every cache manager manages exact information with little management overhead and written data can be buffered in home nodes. We also evaluate and analysis the performance of the home-based Coopc-PVFS in comparison to PVFS and to the hint-based Coopc-PVFS.

This paper is organized as follows. In the next section, we present about PVFS and cooperative cache. In section 3, we describe about Coopc-PVFS and the previous developed hint-based Coopc-PVFS. In section 4, we present the design and implementation of the home-based Coopc-PVFS. In section 5, we evaluate and analysis the performance of the home-based Coopc-PVFS in comparison to PVFS and to the hint-based Coopc-PVFS. Finally, we summarize major contributions of this work and discuss future work in section 6.

\section{Related Work}

\subsection{PVFS (Parallel Virtual File System)}

PVFS consists of compute nodes, a metadata manager and $\mathrm{I} / \mathrm{O}$ servers. The compute nodes are clients that use PVFS services. A metadata manager manages metadata of PVFS files. The I/O servers store actual data of PVFS files. In PVFS, a file data is stripped over I/O servers.

There was a study for the file system caching effect of PVFS. Vilayannur et al. [2] designed and implemented a file system cache of a client. They showed that a file system cache in a client is efficient if many applications in the client share files among them. But their research was limited to a file system cache in a single node.

\subsection{Cooperative Cache}

Cooperative cache $[3,4,5]$ was proposed to reduce servers' load and to get high performance. In cooperative cache, if a file system cache in a client doesn't handle a request to a file, the client sends the request to the other client's cache that caches the file rather than to the server because the access time of another client's memory is faster than that of the server's disk. Servers' load can be reduced in cooperative cache so that it is scalable as the number of clients increases. Because there is much more memory in the cooperative cache than in a single file system cache, the cooperative cache can handle more requests and improve overall system performance.

There have been many studies about cooperative caching. Dahlin et al. [3] suggested the efficient cache management scheme called N-chance algorithm, Feeley et al. [4] suggested another efficient cache management scheme called modified Nchance algorithm in GMS (Global Memory Service). Sarkar et al. [5] suggested the hint-based cooperative caching to reduce the management overhead using hint. Thus, the hint-based cooperative cache is scalable and can be adopted in the large-scale system such as cluster computer. 


\section{Cooperative Cache for PVFS}

\subsection{Overview of Cooperative Cache for PVFS}

In the PVFS, an application reads/writes a file through $\mathrm{I} / \mathrm{O}$ servers without a file system caching facility. We made Coopc-PVFS as we added a cache manager to a PVFS client.

In figure 1, we present the workflow of Coopc-PVFS added to a PVFS client.

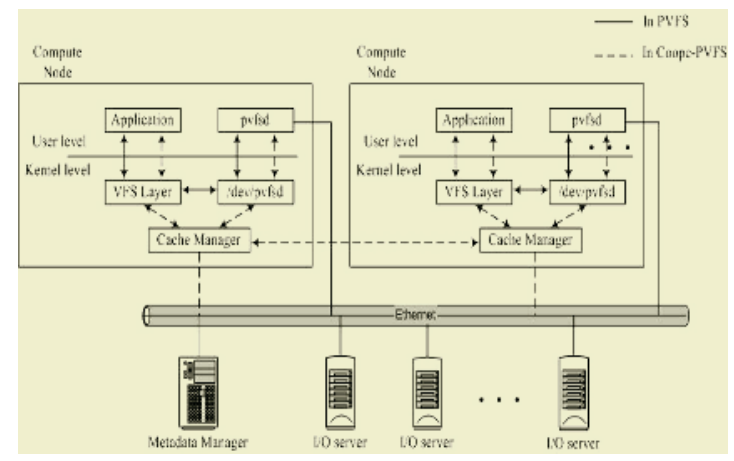

Fig. 1. Workflow of Coopc-PVFS

After we added a cache manager to a PVFS client, a cache manager can cache data and does cooperation with other clients' cache managers.

\subsection{Hint-Based Cooperative Cache for PVFS}

Because of large overhead to maintain accurate information about cached blocks, we designed Coopc-PVFS as a hint-based cooperative cache [9]. To maintain the hint opened clients list, we added new function to the metadata manager to keep the clients list that contains information of clients that opened the file before. Whenever a client opens a file, the client gets both the metadata and the opened clients list of the file from the metadata manager. To accurately look up where is a block, a client must manage information about cached blocks in its own cache and other clients. To maintain this information, each cache manager manages its own bitmap and exchanges its own information with each other when it gets the block from another client.

Unlike previous hint-based cooperative cache research [5], we managed information and cached blocks per block, not per file. Because many clients share large files among them in a parallel file system, it is more adaptable to manage information and to cache per block than per file in Coopc-PVFS.

In PVFS, all the accesses to files go through the $\mathrm{I} / \mathrm{O}$ servers. To conserve the consistency the same as in PVFS, the cache manager must invalidate blocks cached in other clients before writing the block to the I/O server in Coopc-PVFS. To do so, whenever an application writes a block, the cache manager sends cache invalidation messages to the others through the metadata manager before sending the written block to the $\mathrm{I} / \mathrm{O}$ server. 


\section{Design and Implementation of the Home-Based Cooperative Cache for PVFS}

In the home-based Coopc-PVFS, every cached block has its own 'home node'. The home node of a block is statically determined and is not changed forever. When a client reads/writes a file, a cache manager finds a block first in its own cache and sends block request to the home node if a block is not in its own cache. Every $\mathrm{read} /$ write request to a block missed in every client' file system cache goes to the home node so that there is cache locality in the home-based Coopc-PVFS. Cache locality and little information management overhead in the home-based Coopc-PVFS can improve more read performance than the hint-based Coopc-PVFS. Also, a home node can buffer written data from clients and all write operations to a block can be gathered and buffered in a home node. Therefore, the home-based cooperative cache can improve more write performance than the hint-based Coopc-PVFS and other previous cooperative caches - only focused on shared read data - because of write buffering and gathering. In the hint-based Coopc-PVFS, there is much penalty of false information and information management overhead so that read/write performance could be worse than the home-based Coopc-PVFS.

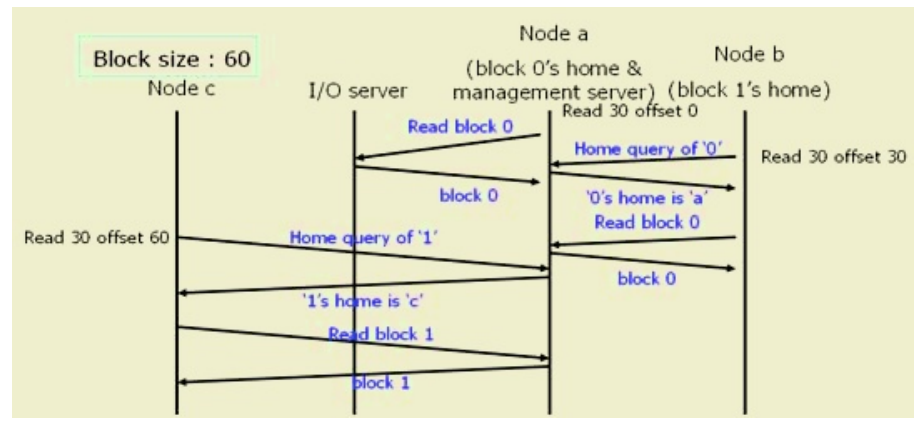

Fig. 2. Message transfers when read operations occur in Home-based Coopc-PVFS

For scalability of home-based Coopc-PVFS, we manage servers per file to reduce home node management overhead: every file has its management server. The management server allocates and manages home nodes of a file. Therefore, we can distribute home node management overhead over nodes. The management server of a file is determined at first file open time in the system and this management server information is saved in the metadata. Therefore, every client can know the management server of a file.

Figure 2 shows message transfers when read operations occur.

When a client reads a block, a cache manager in the client finds a block from its own cache first and read operation is over if there is a block in its own cache. If there is not in its own cache, a cache manager finds a home node of the block. If the home node is not allocated, a cache manager gets the home node from a management server of the file. After knowing the home node, a cache manager gets the block from the home node and caches it so that read operation is over. When a home node gets block 
requests from other nodes, it transfers the block if there is the block in its own cache or it transfers the block to other clients' cache managers after it gets the block from $\mathrm{I} / \mathrm{O}$ servers if there is not. Also, the home node remembers nodes that read the block so that the home node can invalidate all cached blocks when there are write operations to the block.

Figure 3 shows message transfers when write operations occur.

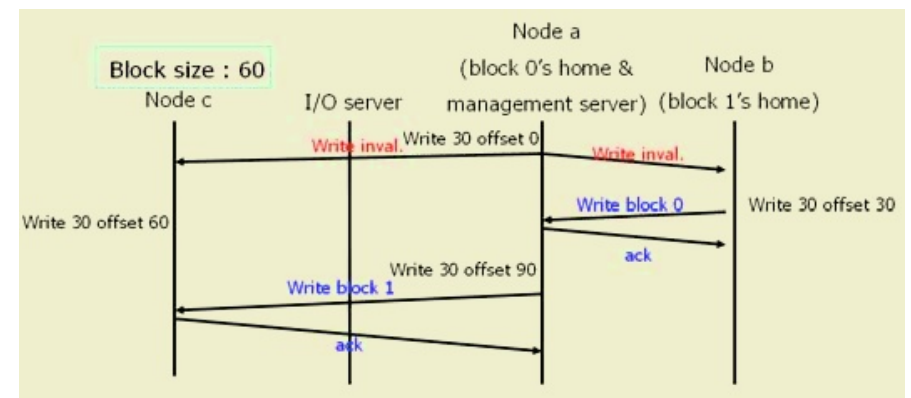

Fig. 3. Message transfers when write operations occur in Home-based Coopc-PVFS

When a client writes a block, a cache manager looks up which node is the home node. If there is no home node, a cache manager gets the home node from a manager node.

If the home node is itself, a cache manager invalidates all cached blocks in others' cache before writing a block and buffers written data to its own cached block. If the home node is another node, a cache manager sends written data to the home node and the home node cache manager invalidates all cached blocks in others' caches before buffering written data to its own cache. Therefore, all written data are buffering in cooperative cache and write performance can be improved in home-based cooperative cache. Also, all written data for a block can be buffering in only one node-home node and all write operations to a block are gathered in the home node so that we can reduce $\mathrm{I} / \mathrm{O}$ servers' overhead.

The home-based Coopc-PVFS is implemented like the previous hint-based cooperative cache. A cache manager is not using Linux page cache system so that a replacement manager in a cache manager manages cached blocks according to the amount of free memory in the system using LRU list. For managing home nodes, a management server per file manages home lists. To manage management servers of files in a metadata manager, we added management servers' lists to a metadata manager. After a client opens a file, a cache manager gets home nodes of a file from a management server. Using this information, a cache manager can read/write blocks to/from home nodes of blocks.

\section{Performance Evaluation}

We used CAN cluster [6] in KAIST to evaluate the performance of Coopc-PVFS. The system configuration of CAN cluster is presented in table 1 . 
Table 1. System configuration

\begin{tabular}{|c|c|}
\hline CPU & Pentium IV 1.8GHz \\
\hline Memory & 512MByte 266MHz DDR \\
\hline Disk & IBM 60G 7200rpm \\
\hline Network & 3c996B-T(Gigabit Ethernet) \\
& 3c17701-ME(24port Gigabit Ethernet Switch) \\
\hline OS , PVFS & Linux(Kernel version 2.4.18), 1.6.0 \\
\hline
\end{tabular}

The metadata manager was allocated in one node and the $\mathrm{I} / \mathrm{O}$ server was allocated in another node. And four other clients were used to execute the test applications -a simple matrix multiplication program and BTIO benchmark programs. Each program operates like below:

! Matrix multiplication program: Each process in four nodes reads each part of two input files of $1024 * 1024$ matrix and does matrix multiplication, and then writes results to a output file. Using the MPI library [8], applications are executed in four nodes.

! BTIO benchmark programs: BTIO is a parallel file system benchmark. It is one of NAS Parallel Benchmark [7]. BTIO contains four programs. In table 2, we present each program. We can evaluate the performance using four nodes with smallest size -class s- in BTIO.

Table 2. BTIO benchmark programs

\begin{tabular}{|l|l|}
\hline Full $($ mpi_io_full $)$ & MPI I/O with collective buffering \\
\hline Simple $($ mpi_io_simple $)$ & MPI I/O without collective buffering \\
\hline Fortran(fortran_io $)$ & Fortran 77 file operations used \\
\hline Epi $\left(e p \_i o\right)$ & $\begin{array}{l}\text { Each process writes the data belonging to its } \\
\text { part of the domain to a separate file }\end{array}$ \\
\hline
\end{tabular}

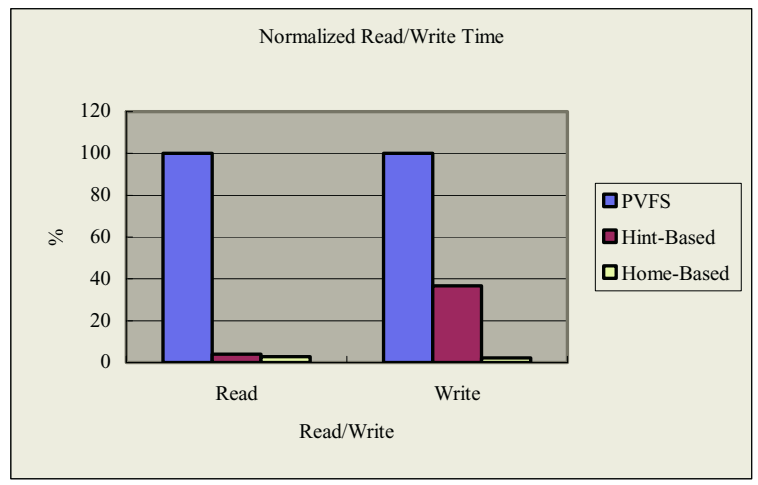

Fig. 4. Normalized read/write time in matrix multiplication program 


\subsection{Execution Time of Matrix Multiplication Program}

In figure4, we present normalized total read/write time based on total read/ write time in PVFS when we execute matrix multiplication program.

Matrix multiplication program is a read-dominant program and total read time in PVFS is about 12 seconds. In the hint-based Coopc-PVFS and in the home-based Coopc-PVFS, total read time is about 0.5 seconds and 0.3 seconds. In this program, read files (input files) are always read and written file (output file) is always written. So, total read time in the home-based Coopc-PVFS is similar to in the hint-based Coopc-PVFS. Total write time in home-based Coopc-PVFS decreases about $94 \%$ of total write time in hint-based Coopc-PVFS because there is no write overhead and home nodes do write-buffering in home-based Coopc-PVFS.

\subsection{Execution Times of BTIO Benchmark Programs}

In figure 5, we present normalized total $\mathrm{read} /$ write time based on total read/write time in PVFS when we execute BTIO benchmark programs.

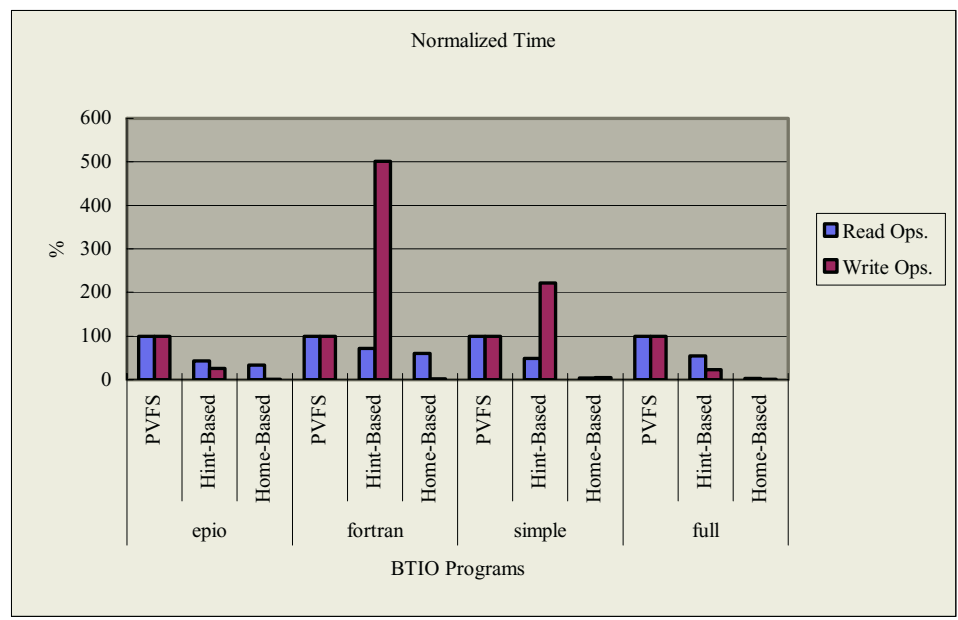

Fig. 5. Normalized read/write time in BTIO benchmark programs

Total read time in the home-based Coopc-PVFS is shorter than in the hint-based Coopc-PVFS and in PVFS because of caching effects and small management overhead. When read/write are all mixed up (fortran and simple cases), total write time in the hint-based Coopc-PVFS is much worst than in PVFS. In all programs, total write time in the home-based Coopc-PVFS is much shorter than in the hintbased Coopc-PVFS and in PVFS because all write operation is buffered in home nodes. 


\section{Conclusion and Future Work}

In this paper, we present the home-based Coopc-PVFS. Also, we evaluate and analysis the performance of the home-based Coopc-PVFS in comparison to PVFS and to the hint-based Coopc-PVFS. The home-based Coopc-PVFS improve read/write performance more than the hint-based Coopc-PVFS. When read operation occurs, a client gets a block just from the home node in the home-based Coopc-PVFS with less overhead than in the hint-based Coopc-PVFS so that there is cache locality in the home-based Coopc-PVFS. When a client write a block, the home-based Coopc-PVFS does write buffering in the home node and gathers all write operations to a block in the home node so that write performance in the home-based Coopc-PVFS is much better than in the Coopc-PVFS and in PVFS.

In the future, we will evaluate the performance of Coopc-PVFS with real parallel programs and various benchmarks. We will do more research about cache management schemes and home node management schemes in the home-based cooperative cache.

\section{References}

[1] P. H. Carns, W. B. Ligon III, R. B. Ross, and R. Thakur, "PVFS: A Parallel File System For Linux Clusters", Proceedings of the 4th Annual Linux Showcase and Conference, Atlanta, GA, October 2000, pp. 317-327

[2] M.Vilayannur,M.Kandemir, A.Sivasubramaniam, "Kernel-Level Caching for Optimizing I/O by Exploiting Inter-Application Data Sharing", IEEE International Conference on Cluster Computing (CLUSTER'02),September 2002

[3] Dahlin, M., Wang, R., Anderson, T., and Patterson, D. 1994. "Cooperative Caching: Using remote client memory to improve file system performance", In Proceedings of the First USENIX Symposium on Operating Systems Design and Implemntation. USENIX Assoc., Berkeley, CA, 267-280

[4] Feeley, M. J., Morgan, W. E., Pighin, F. H., Karlin, A. R., and Levy, H. M. 1995. "Implementing global memory management in a workstation cluster", In Proceedings of the 15th symposium on Operating System Principles (SOSP). ACM Press, New york, NY, 201-212

[5] Prasenjit Sarkar, John Hartman, "Efficient cooperative caching using hints", Proceedings of the second USENIX symposium on Operating systems design and implementation, p.35-46, October 29-November 01, 1996, Seattle, Washington, United States

[6] Can cluster, http://camars.kaist.ac.kr/ nrl

[7] Parkson Wong, Rob F. Van der Wijngaart, NAS Parallel Benchmark I/O Version 2.4, NAS Technical Report NAS-03-002, NASA Ames Research Center, Moffett Field, CA 94035-1000

[8] W. Gropp, S. Huss-Lenderman, A. Lumsdaine, E. Lusk, W. Nitzberg, W. Saphir, M. Snir. MPI: The Complete Reference (vold.2). MIT Press, 1998

[9] In-Chul Hwang, Hojoong Kim, Hanjo Jung, Dong-Hwan Kim, Hojin Ghim, Seung-Ryoul Maeng and Jung-Wan Cho, Design and Implementation of the Coopeartive Cache for PVFS, International Conference on Computational Science 2004, Krakow, Poland, June 2004. 